\title{
Isolation and Characterization of a Theta Glutathione S-transferase Gene from Panax ginseng Meyer
}

\author{
Yu-Jin Kim, Ok Ran Lee ${ }^{1}$, Sungyoung Lee², Kyung-Tack Kim³ ${ }^{3}$ and Deok-Chun Yang ${ }^{1^{*}}$ \\ ${ }^{1}$ Department of Oriental Medicinal Materials and Processing, College of Life Science, Kyung Hee University, \\ Suwon 449-701, Korea \\ ${ }^{2}$ Department of Computer Engineering, Kyung Hee University, Yongin 449-701, Korea \\ ${ }^{3}$ Processing Technology Research Group, Korea Food Research Institute, Seongnam 463-746, Korea
}

\begin{abstract}
Plants have versatile detoxification systems to encounter the phytotoxicity of the wide range of natural and synthetic compounds present in the environment. Glutathione S-transferase (GST) is an enzyme that detoxifies natural and exogenous toxic compounds by conjugation with glutathione (GSH). Recently, several roles of GST giving stress tolerance in plants have demonstrated, but little is known about the role of ginseng GSTs. Therefore, this work aimed to provide further information on the GST gene present in Panax ginseng genome as well as its expression and function. A GST cDNA (PgGST) was isolated from P. ginseng cDNA library, and it showed the amino acid sequence similarity with theta type of GSTs. PgGST in ginseng plant was induced by exposure to metals, plant hormone, heavy metals, and high light irradiance. To improve the resistance against environmental stresses, full-length cDNA of PgGST was introduced into Nicotiana tabacum. Overexpression of PgGST led to twofold increase in GST-specific activity compared to the non-transgenic plants, and the GST overexpressed plant showed resistance against herbicide phosphinothricin. The results suggested that the $\operatorname{Pg} G S T$ isolated from ginseng might have a role in the protection mechanism against toxic materials such as heavy metals and herbicides.
\end{abstract}

Keywords: Panax ginseng, Environmental stress, Glutathione-S-transferase, Phosphinothricin

\section{INTRODUCTION}

Glutathione S-transferases (EC 2.5.1.18) are ubiquitous family of multifunctional proteins that catalyze the conjugation of the tripeptide glutathione (GSH) to large variety of hydrophobic compounds containing electrophilic centers. This reaction yields a GSH conjugate, which is often inactive, water-soluble, and is usually less toxic than the parent compound [1]. In plants, these conjugates are sequestered into the vacuole where they are further processed and detoxified [2]. Most studies are done using mammalian glutathione S-transferases

(c) This is an Open Access article distributed under the terms of the Creative Commons Attribution Non-Commercial License (http://creativecommons.org/licenses/by-nc/3.0/) which permits unrestricted non-commercial use, distribution, and reproduction in any medium, provided the original work is properly cited.
(GSTs), which are categorized into five classes, alpha, $\mathrm{mu}, \mathrm{pi}$, theta, and sigma, based on the amino acid identity, immuno-cross reactivity, and substrate specificity [3]. The theta class is the largest class of GST isozymes with more than 50 members and finds in the widest range of species including bacteria, insects, plants, fish, and mammals. Many GSTs have been purified from animals over the last $30 \mathrm{yr}$ and classified [4], and several plant GSTs have been characterized [2].

GSTs appear to play an important role in protecting

Received 10 Jun. 2012, Revised 11 Aug. 2012, Accepted 11 Aug. 2012

*Corresponding author

E-mail: dcyang@khu.ac.kr

Tel: +82-31-201-2100, Fax: +82-31-205-2688 
plants as well as animals against oxidative damage [5]. Plants often encounter various kinds of environmental stress such as pathogen attack, wounding, heavy metals, and extreme high or low temperatures. In all these processes, a common result is the production of reactive oxygen species (ROS), which would then generate membrane lipid peroxidation and the formation of highly cytotoxic products. GSTs can remove membrane lipid peroxides as well as products of oxidative DNA degradation by conjugating them with GSH [6]. Expression of GSTs in plants is highly responsive to biotic and abiotic stresses and to a wide variety of stress-associated chemicals [7].

Despite the presence of GST genes in various plants, up to now there was no report of any type of GST in ginseng plant. As most of crop plants have to endure various stresses during their life cycle, perennial ginseng plant also faced these kinds of difficulties. Because ginseng is a shade-loving perennial herb, which is cultivated 4 to 5 years, oxidative stress may seriously affect its growth. Ginseng (Panax ginseng Meyer) is one of the most widely used herbal medicines in the Orient. Despite the medicinal importance of ginseng, few antioxidant genes in ginseng plant have studied in molecular levels [8]. The present study aims to provide further information about a cloning of GST gene present in P. ginseng genome using available ginseng expressed sequence tag (EST) library [9], and as well as its expression and functional characteristics against various environmental stresses.

\section{MATERIALS AND METHODS}

\section{Plant materials and growth conditions}

Ginseng ( $P$. ginseng) was collected from the Ginseng Genetic Resource Bank in Korea. Three-week-old ginseng plants were used for gene expression analysis using reverse transcriptase polymerase chain reaction (RT-PCR). Ginseng seeds were in vitro germinated in Murashige and Skoog (MS) media (Duchefa Biocheme, Haarlem, the Netherlands) with $3 \%$ sugar at $25^{\circ} \mathrm{C}$ under a 16-h photoperiod.

\section{Glutathione S-transferase gene sequence and its sequence analysis}

The cDNA library [9] constructed previously was utilized for searching of GST gene in ginseng. Homologous sequences of GST EST were searched against the GenBank databases using a BLASTX algorithm. A pTriplEx phagemid for full-length of GST cDNA was excised from the $\lambda p$ TriplEx 2 and used as templates for sequence analysis. Nucleotide and amino acid sequence analyses were performed using DNASIS program (Hitachi, Tokyo, Japan). These deduced amino acid sequences were searched for homologous proteins in the databases using BLAST network services at the National Center for Biotechnology Information. We used ClustalX with default gap penalties to perform multiple alignment of GST isolated in ginseng and previously registered in other species. The protein property was estimated using ProtParam [10].

\section{Environmental stresses treatment}

To investigate the response of the PgGST against various stresses, ginseng plantlets were treated with abiotic stimuli in about $3 \mathrm{wk}$ after zygotic embryo germination as described previously [11]. For chemical stress or plant hormone treatments, the plantlets were placed for various periods in MS medium containing indicated concentrations of chemicals; $100 \mathrm{mM} \mathrm{NaCl}, 10 \mathrm{mM} \mathrm{H}_{2} \mathrm{O}_{2}, 20 \%$ sucrose, $11 \%$ mannitol, $500 \mu \mathrm{M} \mathrm{CuSO}_{4}, 500 \mu \mathrm{M} \mathrm{CdSO}_{4}$ or $1 \mathrm{mM}$ salicylic acid (SA), $0.2 \mathrm{mM}$ jasmonic acid (JA), $0.1 \mathrm{mM}$ abscisic acid (ABA). Chilling stress was applied by exposing the plantlets at $4^{\circ} \mathrm{C}$. For the UV treatment, the plantlets were irradiated under UVC lamps at 1.35 $\mu \mathrm{E} \mathrm{m}^{-2} \mathrm{~s}^{-1}$ (below $280 \mathrm{~nm}$ ). To monitor the effect of light stress, plants were kept in the white light with intensity of about $500 \mu \mathrm{mol} \mathrm{m} \mathrm{s}^{-1}$. In all cases, stress treatments were carried out on the MS media and 10 plantlets were treated with each stress for $1,4,8,24,48$, or $72 \mathrm{~h}$. The stressed plant materials from all completed treatments were immediately frozen in liquid nitrogen and stored at $-70^{\circ} \mathrm{C}$ until required.

\section{Semi-quantitative reverse transcriptase poly- merase chain reaction analysis}

Total RNA was extracted from different parts of whole plant tissues (leaves, stems, and roots) and seedlings of $P$. ginseng using RNeasy mini kit (Qiagen, Valencia, CA, USA). For RT-PCR, 200 ng of total RNA was used as a template for reverse transcription using oligo $(\mathrm{dT})_{15}$ primer $(0.2 \mathrm{mM})$ and AMV Reverse Transcriptase (10 $\mathrm{U} / \mu \mathrm{L}$; Intron Biotechnology Inc., Seongnam, Korea) according to the manufacturer's instructions. The PCR reaction was then performed using a $1 \mu \mathrm{L}$ aliquot of the first strand cDNA in a final volume of $20 \mu \mathrm{L}$ containing 5 pmol of specific primers for coding region of $P g G S T$ (forward, 5'-CGT TCT CAT CTT CTG CAA GGT C-3'; reverse, 5'-GCT GAG ATC TGC TAT GGA TGG T-3'). As a control, the primers specific to $P$. ginseng actin gene were used (forward, 5'- CGT GAT CTT ACA GAT AGC 
TTG ATG A-3'; reverse, 5'- AGA GAA GCT AAG ATT GAT CCT CC-3'). PCR was carried out using 2X Taq Premix (SolGent Co., Daejeon, Korea) in a thermal cycle programmed as follows: an initial denaturation for $5 \mathrm{~min}$ at $96^{\circ} \mathrm{C}, 30$ amplification cycles, $\left(20 \mathrm{~s}\right.$ at $95^{\circ} \mathrm{C}$ [denaturation], $45 \mathrm{~s}$ at $60^{\circ} \mathrm{C}$ [annealing], and $1 \mathrm{~min}$ at $72^{\circ} \mathrm{C}$ [polymerization]), followed by a final elongation for $10 \mathrm{~min}$ at $72^{\circ} \mathrm{C}$. The actin gene was PCR-amplified in the same PCR conditions as $P g G S T$ with the same amplification cycles (30 cycles), and was used as an internal control to normalize each samples. Seven microliters of the reaction mixture was analyzed on a $1 \%(\mathrm{~W} / \mathrm{V})$ agarose gel containing ethidium bromide in $1 \mathrm{X}$ TAE buffer and then photographed for the expression analysis.

\section{Vector construction and plant transformation}

The entire coding region of GST gene was amplified from $P$. ginseng cDNA using PCR with specific primers embedding $\mathrm{NcoI}$ and $\mathrm{BamHI}$ restriction sites. The constructed PCR product was ligated into the same restriction sites between double $35 \mathrm{~S}$ cauliflower mosaic virus (CaMV) promoter followed by alfalfa mosaic virus enhancer and the nopaline synthase (NOS) terminator in 524Xba cassette vector (NRC Plant Biotechnology Institute, Saskatoon, Canada). The 524Xba recombinant vector carrying the $P g G S T$ was digested with $X b a \mathrm{I}$ and then the expression cassette was ligated into the same restriction sites within pRD400 (Canada). The construct was verified by automated DNA sequencing and then transformed into Agrobacterium tumefaciens strain LBA4404 according to the tri-parental mating method [12]. Leaf disk explants of Nicotiana tabacum cv. Xanthi were cocultured with $A$. tumefaciens LBA4404 containing pRD400 binary vector. Transgenic plants were selected on the basis of their ability to regenerate on the MS medium containing $2 \mathrm{mg} / \mathrm{L}$ benzylaminopurine, $0.2 \mathrm{mg} /$ L 1-naphthaleneacetic acid (NAA), $100 \mathrm{mg} / \mathrm{L}$ kanamycin sulfate, and $500 \mathrm{mg} / \mathrm{L}$ cefotaxime. Upon transfer to MS medium containing $0.2 \mathrm{mg} / \mathrm{L}$ NAA, transgenic seedlings developed into plantlets.

\section{Analysis of transgenic plants}

The presence of neomycin phosphotransferase II (NPTII) (forward, 5'-GAG GCT ATT CGG CTA TGC TG-3'; reverse, 5'-ATG CCA TAG CGG CGA GGG CTA-3'), $P g G S T$, CaMV promoter (forward, 5'-ATT GTG CGT CCC TTA CGT CAG TGG-3'; reverse, 5'ATC GTT GAA GAT GCC TCT GCC G-3'), and NOS terminator (forward, 5'-GCG GAG AAT TAA GGG AGT CAC G-3'; reverse, 5'-GGA TAC CGA GGG GAA
TTT ATG G-3') transgenes were analyzed by PCR from regenerated leaf tissue samples. For control, internal transcribed spacer primers (forward, 5'-TAC CGA TTG AAT GGT CCG-3'; reverse, 5'-ATA TGC TTA AAC TCA GCG GGT-3') were used for PCR analysis. In addition, Southern blot analysis was carried out to confirm the successful insertion of PgGST into genome of transgenic $N$. tabacum plants. Southern blotting involved digesting 20 $\mu \mathrm{g}$ of leaf genomic DNA with EcoRI and HindШ, then electrophoresing the digested products on a 1.3\% agarose gel (50 V, overnight). DNA was transferred to a nylon membrane using a Turbo blotter apparatus (Schleicher and Schuell, Maidstone, UK). Labeling of the probes, hybridization, and detection was carried out using DIG High Prime DNA Labeling and Detection Starter Kit following the instruction given by the manufacturer (Roche, Mannheim, Germany).

\section{Analysis of transcripts of transgenic plant against chemical stresses}

Differential transcriptional level against chemical stresses was carried out by RT-PCR using gene-specific primers. As a loading control, actin gene was amplified using actin specific primers (forward, 5'-GAA CGG GAA ATT GTT CGA GA-3'; reverse, 5'-GCA GAT TCC ATT CCG ATC AT-3'). For stress treatment, we sprayed on tobacco seedlings with distilled water containing each of $100 \mu \mathrm{M} \mathrm{CdSO}, \mathrm{CuSO}_{4}, \mathrm{ZnSO}_{4}$ or phosphinothricin (PPT), and $1 \mathrm{mM}$ 2,4-D or SA. After $4,12,24$, and $48 \mathrm{~h}$, respectively, treated leaves were immediately frozen in liquid nitrogen and stored at $-70^{\circ} \mathrm{C}$. Total RNA was isolated using RNeasy mini kit (Qiagen, Valencia, CA, USA), and RT-PCR was conducted by the method described above.

\section{Glutathione S-transferase activity assay}

GST activity was determined according to the procedure described by Dean et al. [13]. The substrates 1-chloro-2,4 dinitrobenzene (CDNB) and reduced GSH were used to measure GST activity. Leaf samples were homogenized by grinding with sodium phosphate buffer and then centrifuged at $12,000 \mathrm{rpm}$ for $10 \mathrm{~min}$ at $4^{\circ} \mathrm{C}$. The supernatant was used for enzyme activity assay. The reaction mixture contains final concentrations of $20 \mathrm{mM}$ reduced GSH and $20 \mathrm{mM} \mathrm{CDNB}$ which are dissolved in $2.5 \%$ ethanol and potassium phosphate butter $(0.1 \mathrm{M}$, $\mathrm{pH}$ 6.5). GST activity was determined by absorbance readings at $340 \mathrm{~nm}$ using an extinction coefficient of 9.6 $\mathrm{mM}^{-1} \mathrm{~cm}^{1}$. 


\section{Treatment of herbicide phosphinothricin and analysis of chlorophyll contents}

To treat PPT, leaves of 3-week-old tobacco plantlets cultivated in a controlled growth chamber were analyzed. Leaf discs (6 $\mathrm{mm}$ diameter), prepared from the leaves of control and transgenic plants, were floated on petri dishes containing $17 \mathrm{~mL}$ of $0.1,1,10,100$, or 1,000
mM PPT solutions. Each treatment was replicated three times, and each replicate consisted of five leaf discs $(6$ $\mathrm{mm}$ diameter) explants. After incubating in the light for 4 and $24 \mathrm{~h}$ at $25^{\circ} \mathrm{C}$, they were ground in liquid nitrogen using a mortar and pestle, and samples were extracted with $700 \mu \mathrm{L}$ of $85 \%(\mathrm{v} / \mathrm{v})$ acetone. Extracts were centrifuged for $15 \mathrm{~min}$ at 3,000 rpm. Chlorophyll contents in

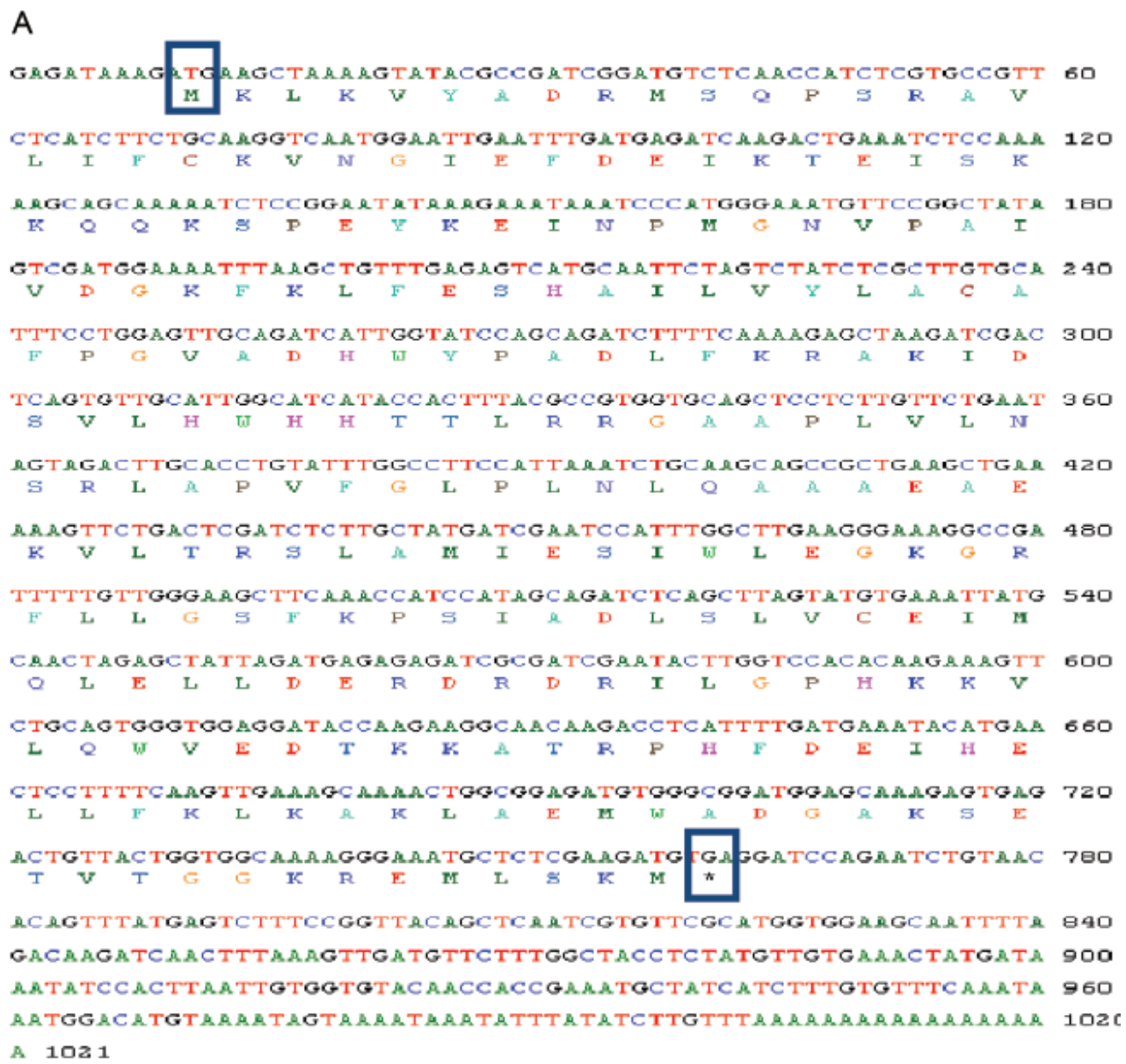

B R L St

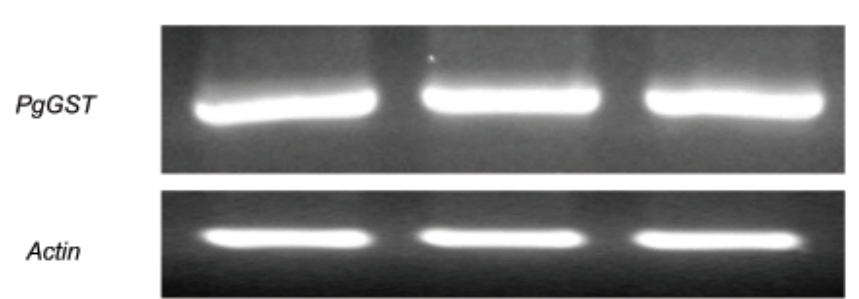

Fig. 1. PgGST sequence analysis. (A) nucleotide and deduced amino acid sequence of a PgGST cDNA isolated from Panax ginseng. The deduced amino acid sequence is shown in single-letter code below the nucleotide sequence with the open reading frame from 10 to 763 bp. The position of nucleotides is shown on the right. Black box shows the transcription start codon and termination codon. The sequence has been deposited in GenBank as accession no. EU625298. (B) Expressions of PgGST in various organs of $P$. ginseng. Total RNAs were extracted from leaves (L), root (R), and stems (St). The actin gene of $P$. ginseng was used as a control. 
the supernatants were measured spectrophotometrically according to the method described previously [14]. The following equations were used for the calculation of chlorophyll a, chlorophyll $\mathrm{b}$, and total chlorophyll. Total chlorophyll=chlorophyll a $[10.3 \times$ E663-0.918 $\times$ E644 (ug/mL)]+chlorophyll b [19.7×E644-3.870×E663 (ug/ $\mathrm{mL})$ ] where, $\mathrm{E}$ is equal optical density at the given wave length.

\section{RESULTS}

\section{Isolation and amino acid sequence analysis of PgGST}

We identified a cDNA clone encoding a GST gene from our EST cDNA library [9] prepared with the taproot of $P$. ginseng. We named this gene as $P g G S T$ with a GenBank accession number EU625298. The PgGST cDNA was 1,021 nucleotides long and had the putative
A

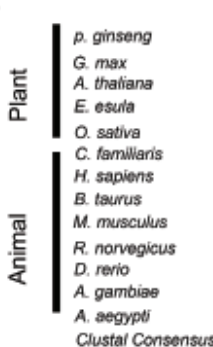

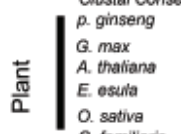

O. sativa
C. familianis

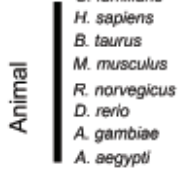

Clustal Consensus

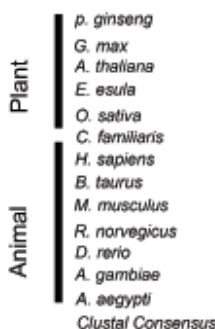

10
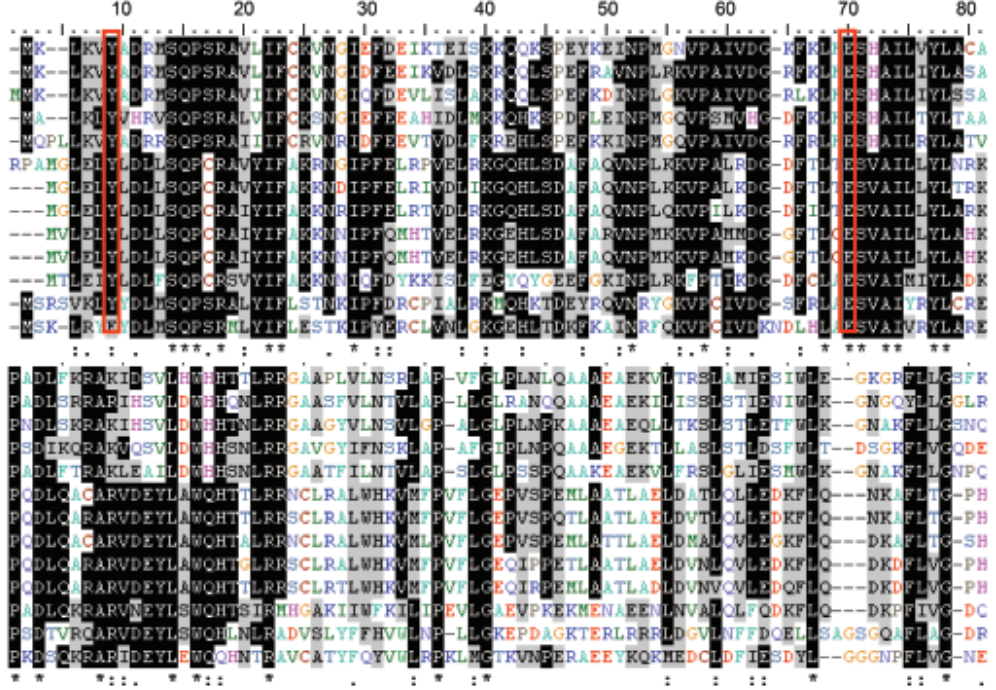

190

30

40

$50 \quad 60$

$70 \quad 80$
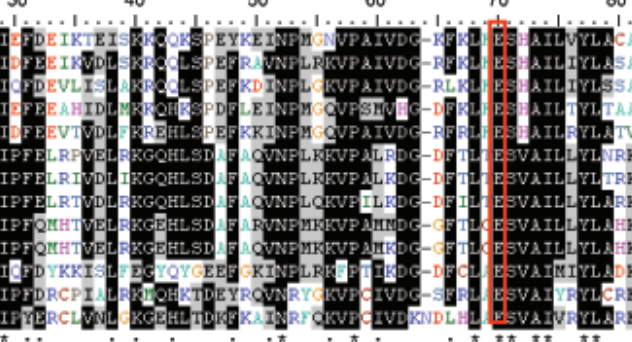

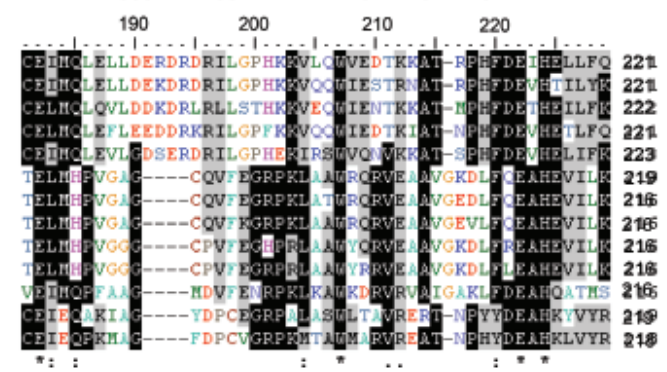

B

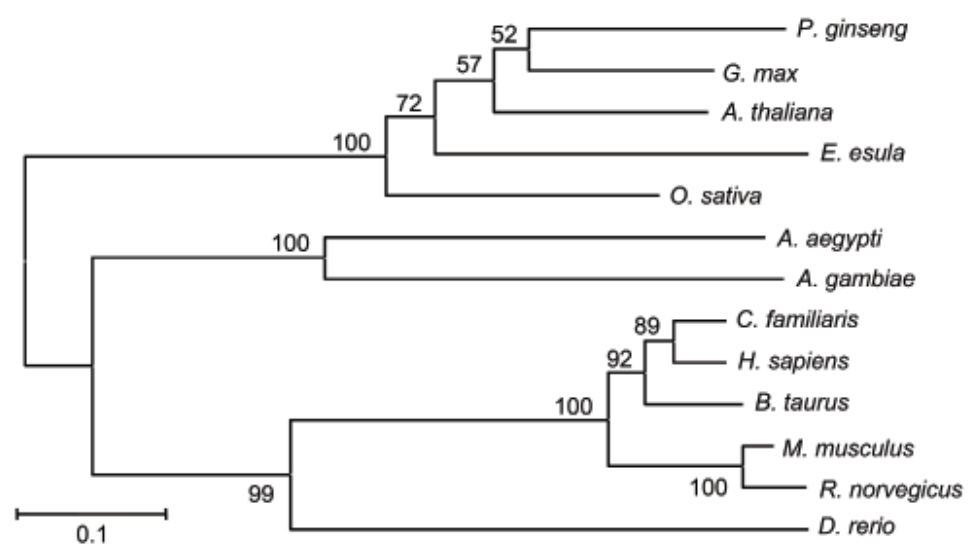

(Continuing) 


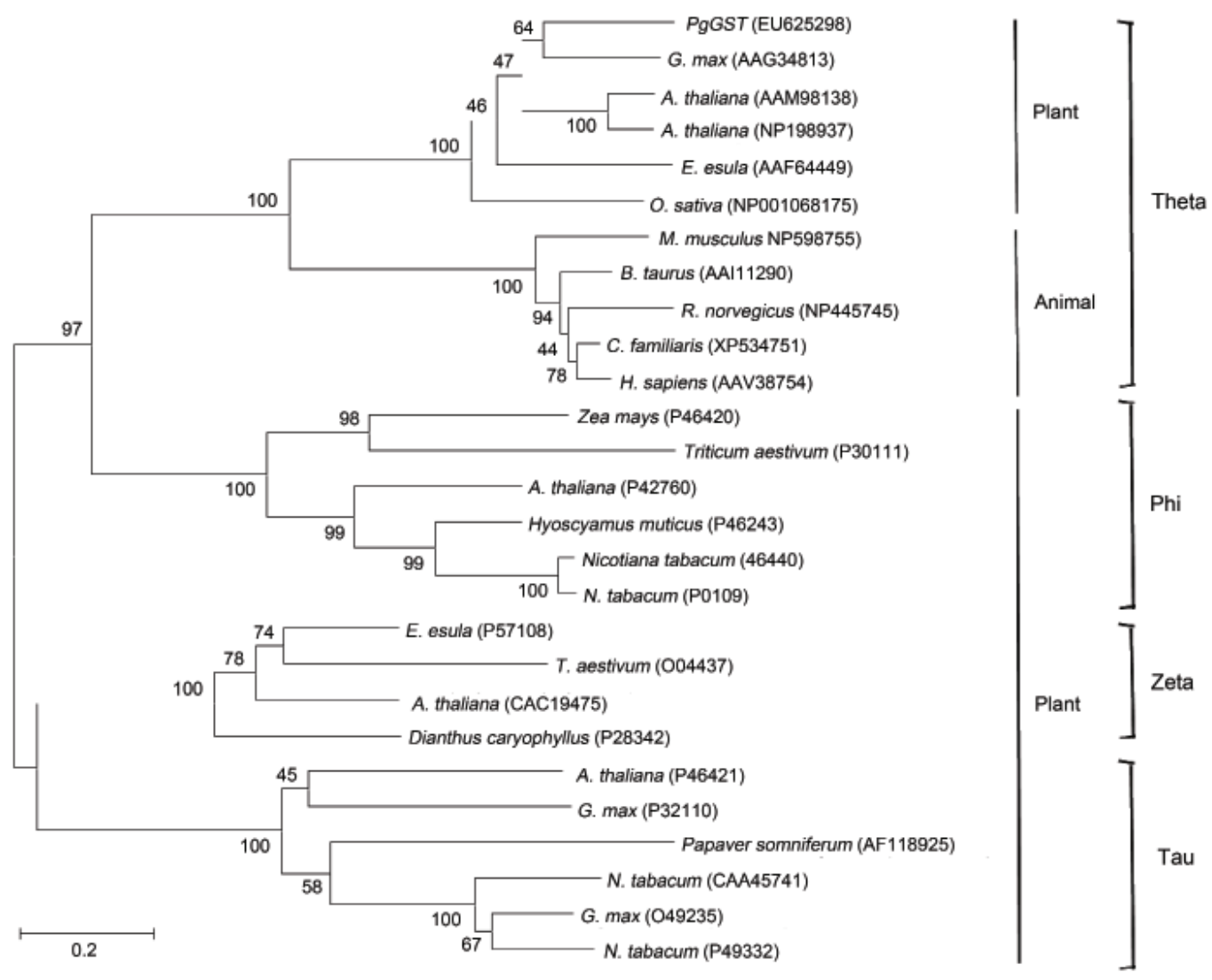

Fig. 2. Sequence homology and phylogenetic analysis of PgGST with other glutathione S-transferase (GST) proteins. (A) comparison of the putative amino acids sequence of PgGST with those of GST genes of theta class from other species with GenBank accession no. in parenthesis; Glycine max (AAG34813), Arabidopsis thaliana (AAM98138), Euphorbia esula (AAF64449), Oryza sativa (AAK98534), Canis familiaris (XP534751), Homo sapiens (AAV38754), Bos taurus (AAl11290), Mus musculus (NP598755), Rattus norvegicus (NP445745), Danio rerio (AAH56725), Anopheles gambiae (AAM61892), and Aedes aegypti (AAV68399). Hyphen was inserted within amino acid sequence to denote gap. Shadow box means well conserved residues, * represents conserved amino acid and, : represents very similar amino acid. Glutathionebinding residues are indicated by red boxes. (B) Phylogeny of the theta class GST protein family from Panax ginseng and other species. Phylogenetic analysis is based on the deduced amino acid sequences of GST genes from various species. Neighbor-joining method was used and the branch lengths are proportional to divergence, with the scale of 0.1 representing $10 \%$ changes.

open reading frame of $753 \mathrm{bp}$, and this ORF encodes a GST protein of 251 amino acids (Fig. 1A). Aliphatic index, which may be regarded as a positive factor for the increase of thermostability, was calculated as 96.80 and instability index is computed to be 41.40 by using ProtParam, and this result means $P g G S T$ classifies as unstable [10]. The calculated molecular mass of the matured protein is approximately $28.3 \mathrm{kDa}$ with a predicated isoelectric point of 9.11. By calculating the hydropathy value by the method of Kyte and Doolittle [15], PgGST was estimated to be hydrophobic. The ubiquitous expression patterns of $P g G S T$ in different organs, leaf, stem and root, were examined using RT-PCR analysis (Fig. 1B).

\section{Phylogenetic analysis}

An amino acid sequence comparison with other known GSTs led to the conclusion that PgGST belongs to subgroup theta (Fig. 2). The putative protein encoded by $P g G S T$ shares high degrees of homologies with Glycine max (AAG34813, 74.2\% identity), Arabidopsis thaliana (AAM98138, 71.4\% identity), Oryza sativa (AAK98534, $66.3 \%$ identity), and Euphorbia esula (AAF64449, $65.1 \%$ identity). Alignment of the GST theta family indicated that amino-acid residues in the N-terminal region are highly conserved, while the $\mathrm{C}$-terminal region shares lower degrees of homology (Fig. 2A). Putative conserved glutathione-binding residues are found (Fig. 2A, redboxed) [3]. Phylogenetic tree of $P g G S T$ with other ho- 


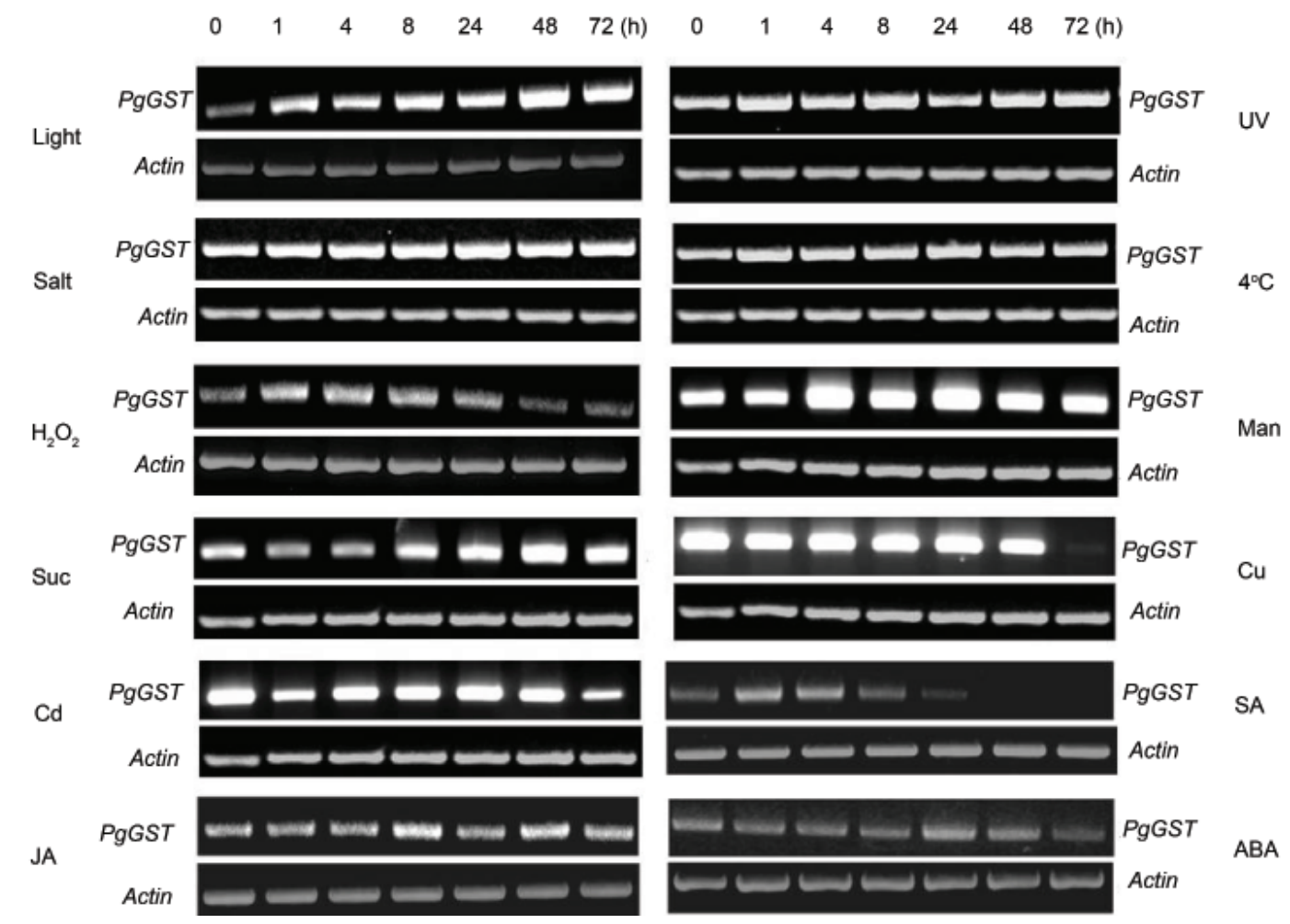

Fig. 3. Reverse transcriptase polymerase chain reaction (RT-PCR) analysis for the expression of PgGST genes in Panax ginseng at various time points (h) post-treatment with various stresses including high light, UV, $100 \mathrm{mM} \mathrm{NaCl}$, chilling $\left(4^{\circ} \mathrm{C}\right), 10 \mathrm{mM} \mathrm{H} \mathrm{O}_{2}, 11 \%$ mannitol (Man), $20 \%$ sucrose (Suc), $500 \mu \mathrm{M} \mathrm{CuSO}_{4}(\mathrm{Cu}), 500 \mu \mathrm{M}$ CdSO4 (Cd), $5 \mathrm{mM}$ salicylic acid (SA), $200 \mathrm{mM}$ jasmonic acid (JA), or $0.1 \mathrm{mM}$ abscisic acid (ABA). A RT-PCR employing actin specific primer was carried out to confirm equal RNA loading.

mologous proteins reveals the closest amino acid identity (Fig. 2B).

\section{Differential expression of PgGST in response to various stresses}

A high light stress caused a rapid induction of $P g G S T$ within 1 h (Fig. 3), whereas $P g G S T$ was not responsive against UV. In response to salinity and chilling treatment, $P g G S T$ transcript level maintained at a steady state until $72 \mathrm{~h}$. Exogenous application of $\mathrm{H}_{2} \mathrm{O}_{2}$ caused a strong induction immediately $(1 \mathrm{~h})$, but transcription was gradually reduced from $4 \mathrm{~h}$ treatment. Under mannitol stress, $P g G S T$ transcription level was increased at $4 \mathrm{~h}$ and was the highest at $24 \mathrm{~h}$ post-treatment. At a sucrose treatment, $P g G S T$ expression was weak at $4 \mathrm{~h}$ treatment, then increased and strongest at $48 \mathrm{~h}$ post-treatment. After copper stress treatment, the PgGST expression continued to be strong from 4 to $24 \mathrm{~h}$. However, a 3-day copper exposure caused a clear decrease in the expression of PgGST. In the case of cadmium stress, its expression was reduced at $1 \mathrm{~h}$, and remained at a slightly increased level until 2 $\mathrm{d}$, then decreased after $3 \mathrm{~d}$. SA caused induction in the expression of PgGST mRNA at an immediate time (1 $\mathrm{h}$ ), and then, its expression was continuously decreased after $8 \mathrm{~h}$ treatment. When continuous SA treatment was maintained, PgGST expression was decreased and no transcripts were observed at 2 to 3 days treatment. JA caused its upregulation at 8 and $48 \mathrm{~h}$ treatment. Under ABA treatment, it seems that $P g G S T$ transcription was strongly expressed at $24 \mathrm{~h}$ post-treatment and was kept decreased in other time zones.

\section{Overexpression PgGST showed enhanced glutathi- one S-transferase activity and resistance against phosphinothricin}

Tobacco was transformed using A. tumefaciens with the $P g G S T$, under the control of CaMV promoter and NOS terminator (PgGSTox). Several independent transgenic lines were established by selection on kanamycin (Fig. 4). Kanamycin resistant callus was developed on the surfaces of explants during the subculture on selection medium. Upon transfer to MS medium containing $0.1 \mathrm{mg} / \mathrm{L}$ NAA, transgenic seedlings developed plantlets. The integration of the PgGST, NPTII, CaMV promoter, 

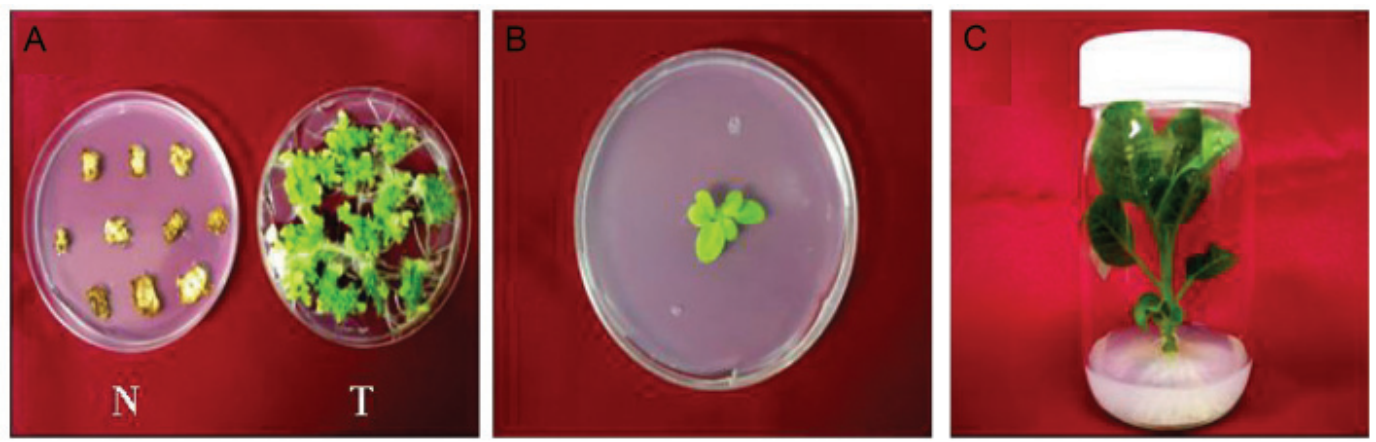

Fig. 4. Genetic transformation of Nicotiana tabacum with ginseng glutathione S-transferase gene. Survived transgenic $(T)$ calli compared with non-transgenic line (N) (A) on selection medium containing $100 \mathrm{mg} / \mathrm{L}$ kanamycin, and $500 \mathrm{mg} / \mathrm{L}$ cefotaxime produced resistant shoot (B), and transgenic tobacco explants acclimated in vitro (C).

A
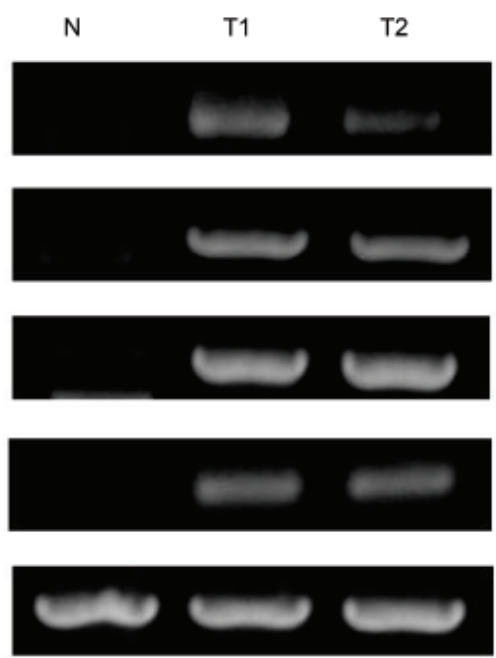

ITS
$\mathrm{C}$

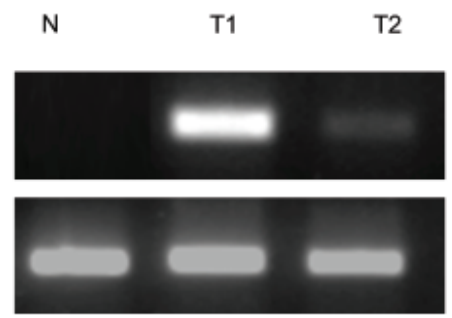

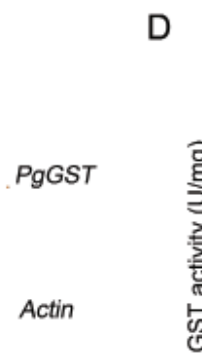

B

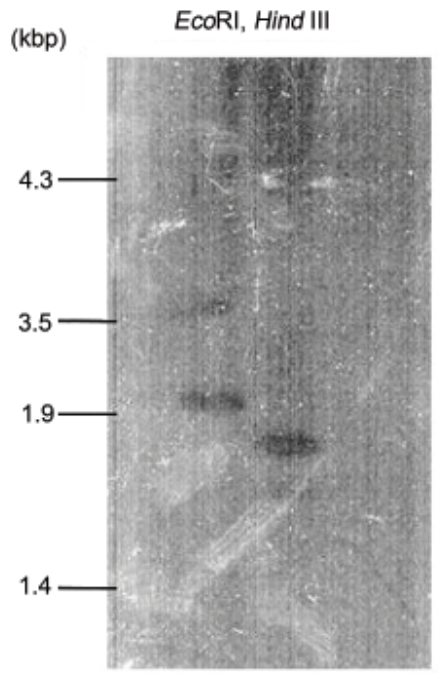

CaMV

PgGST

NPTII

NOS

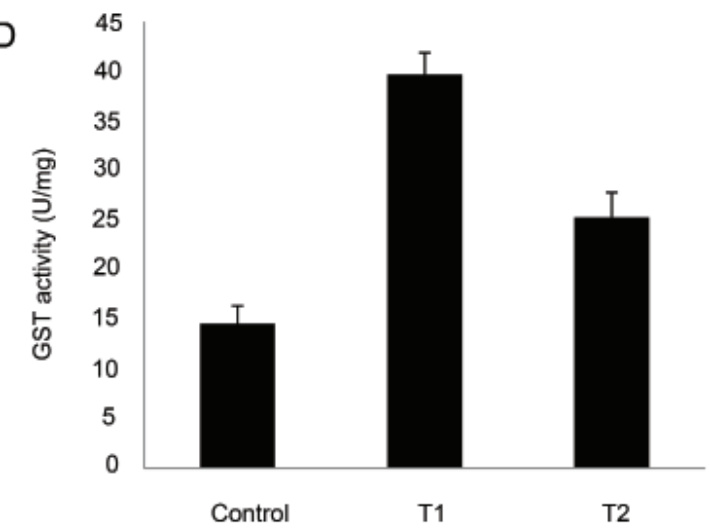

Fig. 5. Confirmation of transgenes in tobacco by polymerase chain reaction (PCR) and southern blot analysis. (A) PCR analysis of $35 S$ cauliflower mosaic virus promoter (CaMV), glutathione S-transferase of Panax ginseng (PgGST), neomycin phosphotransferase II (NPTI), nopaline synthase terminator (NOS), and internal transcribed spacer (ITS) gene as control from non-transgenic (N) and two transgenic tobacco lines (T1 and T2) was conducted. (B) Southern blot hybridization of DNA prepared from transgenic tobacco plants. DNA ( $20 \mu \mathrm{g})$ was cut by restriction enzymes EcoRI or HindIII, separated on 1.3\% agarose gel, transferred to a membrane and hybridized to a DIG-labeled PgGST-specific probe. (C) reverse transcriptase-PCR and (D) glutathione S-transferase (GST) activity of non-transgenic (N) and two transgenic lines (T1 and T2). 
A

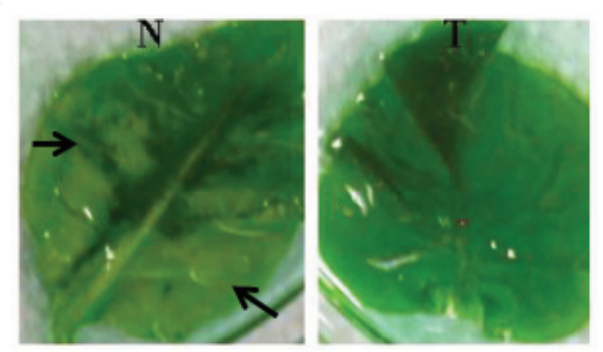

B

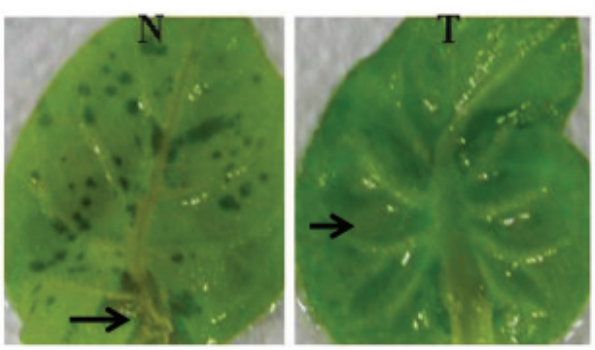

Fig. 6. Leaves of non-transgenic $(N)$ and transgenic ( $T$ and $T 2$ line) tobacco plants treated with $0.1 M(A)$ or $1 \mathrm{M}(B)$ phosphinothricin for $4 \mathrm{~h}$.

A

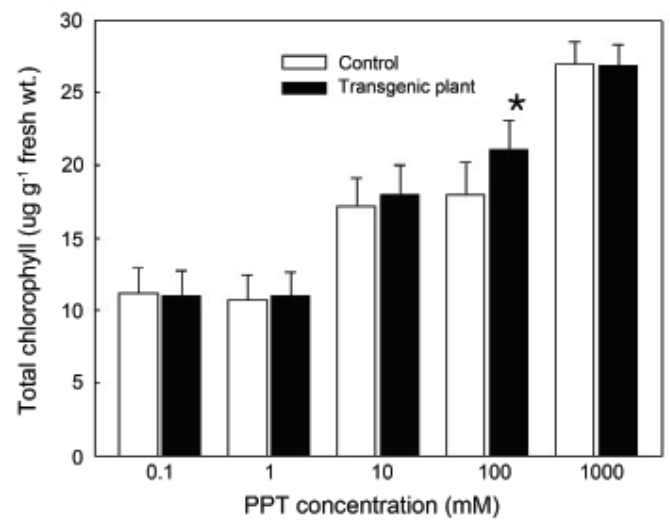

B

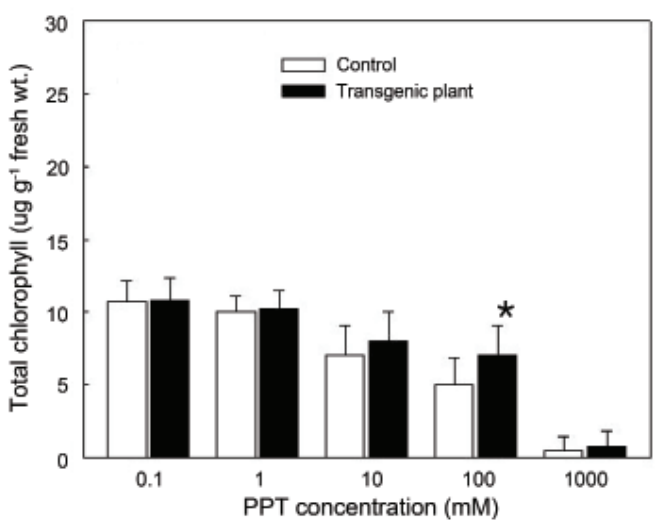

C

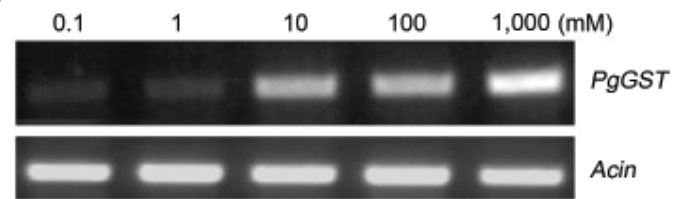

Fig. 7. Effect of phosphinothricin (PPT) on the chlorophyll content and PgGST expression in transgenic tobacco leaves (T2). Total chlorophyll content from leaf discs of wild-type and transgenic plants treated with $0.1,1,10,100$, or 1,000 mM of PPT for $4 \mathrm{~h}(\mathrm{~A})$ and $24 \mathrm{~h}(\mathrm{~B})$ was analyzed. Means $\left( \pm\right.$ SE) are for 10 replicates per treatment. Averages for treated samples were significantly different compared to the control at ${ }^{*} p<0.01$. PgGST expression by PPT $(0.1,1,10,100$, or $1,000 \mathrm{mM})$ for $4 \mathrm{~h}$ was performed by reverse transcriptase polymerase chain reaction (C).

and NOS terminator genes were confirmed by PCR analysis (Fig. 5A). To determine the number transgene in the tobacco genome, we performed a Southern blot analysis. When probed under high-stringency conditions, a single band was detected from genomic DNA after digestion with EcoRI or HindШ (Fig. 5B). RT-PCR analysis and GST activity were assayed in transgenic and wild-type plants. Transgenic $P g G S T$ gene was expressed highly in transgenic tobacco lines (Fig. 5C) and GST-expressing transgenic lines had an approximately twofold higher activity in leaf extracts compared to the control (Fig. 5D). These results confirmed that $P$. ginseng GST transgene encode an active GST in transgenic $N$. tabacum plants.

Generally reported GST-mediated herbicide detoxification suggested us to test its functional resistance against
PPT. Fig. 6 shows that the leaf damage of transgenic (T, $\mathrm{T} 2$ line) and non-transgenic $(\mathrm{N})$ plants when they were treated with $0.1 \mathrm{M}$ (Fig. 6A) and $1 \mathrm{M}$ (Fig. 6B) of PPT for $4 \mathrm{~h}$. The necrotic area in the control leaf is much larger than in the transgenic plants.

Since chlorophyll content is one of well-known indication to access plant damage after stress treatment, its content was analyzed. Chlorophyll contents of tobacco treated with high concentration of PPT at $4 \mathrm{~h}$ treatment were higher than those at low concentration (Fig. 7A). Increased chlorophyll content was correlated with increased transcript level of PgGST (Fig. 7C). Chlorosis developed throughout the leaves according to the treatment time prolonged. Chlorophyll content at $24 \mathrm{~h}$ after PPT treatment was decreased as PPT concentra- 
tion increased (Fig. 7B). At $100 \mathrm{mM}$ PPT, control plant showed significantly lowered total chlorophyll level than transgenic plant (T2). However, higher concentrations of PPT (1 M) application resulted in chlorosis and extensive necrosis in both control and transgenic plants within $24 \mathrm{~h}$ after treatment.

\section{DISCUSSION}

We describe for the first time the isolation and characterization of GST gene in $P$. ginseng. A cDNA encoding a GST, designated $P g G S T$ was isolated and sequenced. Genbank BlastX search, phylogenetic study, and analysis of protein confirmed that the $P g G S T$ should be theta group of GST. The calculated size of $P g G S T$ is $28.3 \mathrm{kDa}$, which is approximately the same size with other reported theta class GSTs [16]. Hydropathy value can explain that $P g G S T$ easily interact with hydrophobic molecules. Each GST has known to contain an independent catalytic site composed of two components. One is a binding site for GSH (G-site) and the other is a site that binds the hydrophobic substrate (H-site) [2]. Alignment of the GST theta family indicated that amino-acid residues in the amino-terminal region are highly conserved, while the $\mathrm{C}$ terminal region shares lower degrees of homology (Fig. 2). One explanation is that the G-site is highly specific for glutathione, which formed a conserved group, and the H-site interacts with electrophilic and hydrophobic xenobiotic substrates, which is much more structurally variable [17]. Although the theta class is the largest class of GST isozymes and its sequence is diverse, several strict sequence conservations are present to distinguish it from the other classes. In the region considered crucial for glutathione binding, a conserved glutamine residue (Q) is present in the other GSTs, which is shown to interact directly with GSH [4]. On the other hand, at this same position of the theta class, a conserved glutamic acid (E) is present instead [3]. A second essential difference between the theta class and the other four classes is a conserved tyrosine residue $(\mathrm{Y})$ near the amino-terminal end that is part of the active site and is located within hydrogen bonding distance of the thiol group of bound GSH. PgGST also has the same residues in the G-site domain as other theta class (Fig. 2).

Plant GST genes exhibit a diverse expression patterns during biotic and abiotic stresses. It becomes obvious that unique combinations of multiple, often interactive signaling pathways from various phytohormones and ROS render the distinct transcriptional activation patterns of individual GSTs during stress [18]. Droog et al. [3] reported that the activity of GST is needed under many different conditions. In case of ginseng GST, it was not significantly responsive against UV, which is distinctly different comparing to the case of GST from Petroselinum crispum where the transcript was induced within $2 \mathrm{~h}$ by UV irradiation [19]. However, PgGST showed significant upregulation by $\mathrm{H}_{2} \mathrm{O}_{2}$ (Fig. 3). Arabidopsis GST gene was also reported to be induced by $\mathrm{H}_{2} \mathrm{O}_{2}$ [20]. GSTs participate in the detoxification of products formed by oxidative damage and thereby protect cells against such stresses [2]. ROS can affect cell viability by causing enzyme inactivation, therefore extensive treatment of $\mathrm{H}_{2} \mathrm{O}_{2}$ could reduce $P g G S T$ expression. In this study, expression of $P g G S T$, which belongs theta class, was not significantly changed against chilling and salinity stresses, whereas tobacco overexpressing tau class of GST was tolerant to low temperature and salt stress exposure [21]. Compatible solutes, such as sucrose and mannitol, are demonstrated to more or less closely relate to antioxidant or osmoprotectant mechanisms [22]. Following similar mechanism, PgGST expression under these compatible solutes was also induced. Expression pattern of PgGST against heavy metals were corresponding with that of theta class GST (GST-theta) from Neanthes succinea where the upregulation of GST-theta was concentration and time dependent [16]. Stress-induced plant growth regulators like ABA, JA, or SA differentially activate GST gene expression [18]. When there was a continuous SA treatment, $P g G S T$ expression was decreased, which can be explained by the role of SA. SA induces cell death via the hypersensitive response, and recent studies have suggested that GSTs act as potential regulators of apoptosis [2].

Previous studies indicated that many theta class members are related with herbicide detoxification. Herbicide detoxification activity was shown for most of theta class subunits [3]. A wheat theta GST gene was found to be closely related to the herbicide-detoxifying GSTs [6], and transgenic tobacco plants overexpressing GST were tolerant to herbicide exposure [21]. Therefore, transgenic tobacco plants were continuously studied to find the evidence of the increasing tolerance against herbicide. PPT, an herbicide commonly used for weed control in agriculture, which is a structural analog of glutamate that binds to glutamine synthetase (GS), a key enzyme of nitrogen metabolism in plants. It leads to the accumulation of ammonia, leaf chlorosis, and an inhibition of photosynthesis [23]. When chlorophyll content was determined from leaves of transgenic and control tobacco plants treated with PPT, interestingly, chlorophyll contents was 
increased by $4 \mathrm{~h}$ treatment of PPT. (Fig. 7A). This result can be explained with previous study that large subunit of Rubisco (LSU) increased after $4 \mathrm{~h}$ PPT treatment to overcome PPT-mediated inhibition of GS [24]. It was suggested that LSU accumulation could be considered as a mechanism to recover from amino acid depletion and ammonia accumulation, when photosynthesis is inhibited by PPT.

The increased $P g G S T$ expression according to the PPT concentration suggests that enhanced GST expression could offer resistance against PPT exposure (Fig. 7). Overall, our results show that the PgGST may have a role in the protection against toxic material such as several heavy metals and herbicides. Further studies are still needed to understand the physiological function of GST in $P$. ginseng. Ginseng transformant might have possibility to endure under the sun light and against heavy metal and herbicide.

\section{ACKNOWLEDGEMENTS}

This research was supported by the Ministry of Knowledge Economy, Korea, under the Information Technology Research Center support program supervised by the National IT Industry Promotion Agency (NIPA2011-C1090-1121-0003).

\section{REFERENCES}

1. Droog F, Hooykaas P, Van Der Zaal BJ. 2,4-Dichlorophenoxyacetic acid and related chlorinated compounds inhibit two auxin-regulated type-III tobacco glutathione S-transferases. Plant Physiol 1995;107:1139-1146.

2. Dixon DP, Lapthorn A, Edwards R. Plant glutathione transferases. Genome Biol 2002;3:REVIEWS3004.

3. Droog F. Plant glutathione S-transferases, a tale of theta and tau. J Plant Growth Regul 1997;16:95-107.

4. Wilce MC, Parker MW. Structure and function of glutathione S-transferases. Biochim Biophys Acta 1994;1205:118 .

5. Park MS, Cho EJ, Lee SK, Lee EJ, Lee DS, Lee KH, Jeon $\mathrm{BH}$. Korean red ginseng protects oxidative injury caused by lead poisoning. J Ginseng Res 2010;34:132-137.

6. Dudler R, Hertig C, Rebmann G, Bull J, Mauch F. A pathogen-induced wheat gene encodes a protein homologous to glutathione-S-transferases. Mol Plant Microbe Interact 1991;4:14-18.

7. Marrs KA. The functions and regulation of glutathione $\mathrm{S}$ transferases in plants. Annu Rev Plant Physiol Plant Mol Biol 1996;47:127-158.
8. Lee OR, Sathiyaraj G, Kim YJ, In JG, Kwon WS, Kim $\mathrm{JH}$, Yang DC. Defense genes induced by pathogens and abiotic stresses in Panax ginseng C.A. Meyer. J Ginseng Res 2011;35:1-11.

9. Kim MK, Lee BS, In JG, Sun H, Yoon JH, Yang DC. Comparative analysis of expressed sequence tags (ESTs) of ginseng leaf. Plant Cell Rep 2006;25:599-606.

10. Gasteiger E, Hoogland C, Gattiker A, Duvaud S, Wilkins MR, Appel RD, Bairoch A. Protein identification and analysis tools on the ExPASy server. In: Walker JM, ed. The proteomics protocols handbook. Totowa: Humana Press, 2005. p.571-607.

11. Kim YJ, Shim JS, Krishna PR, Kim SY, In JG, Kim MK, Yang DC. Isolation and characterization of a glutaredoxin gene from Panax ginseng C. A. Meyer. Plant Mol Biol Report 2008;26:335-349.

12. Ditta G, Stanfield S, Corbin D, Helinski DR. Broad host range DNA cloning system for gram-negative bacteria: construction of a gene bank of Rhizobium meliloti. Proc Natl Acad Sci U S A 1980;77:7347-7351.

13. Dean JV, Gronwald JW, Eberlein CV. Induction of glutathione s-transferase isozymes in sorghum by herbicide antidotes. Plant Physiol 1990;92:467-473.

14. Lichtenthaler HK. Chlorophylls and carotenoids: pigments of photosynthetic biomembranes. Methods Enzymol 1987;34:350-382.

15. Kyte J, Doolittle RF. A simple method for displaying the hydropathic character of a protein. J Mol Biol 1982;157:105-132.

16. Rhee JS, Lee YM, Hwang DS, Won EJ, Raisuddin S, Shin $\mathrm{KH}$, Lee JS. Molecular cloning, expression, biochemical characteristics, and biomarker potential of theta class glutathione S-transferase (GST-T) from the polychaete Neanthes succinea. Aquat Toxicol 2007;83:104-115.

17. Choi CY, An KW, An MI. Molecular characterization and mRNA expression of glutathione peroxidase and glutathione S-transferase during osmotic stress in olive flounder (Paralichthys olivaceus). Comp Biochem Physiol A Mol Integr Physiol 2008;149:330-337.

18. Moons A. Regulatory and functional interactions of plant growth regulators and plant glutathione S-transferases (GSTs). Vitam Horm 2005;72:155-202.

19. Loyall L, Uchida K, Braun S, Furuya M, Frohnmeyer H. Glutathione and a UV light-induced glutathione Stransferase are involved in signaling to chalcone synthase in cell cultures. Plant Cell 2000;12:1939-1950.

20. Chen W, Singh KB. The auxin, hydrogen peroxide and salicylic acid induced expression of the Arabidopsis GST6 promoter is mediated in part by an ocs element. Plant J 1999;19:667-677. 
21. Roxas VP, Smith RK Jr, Allen ER, Allen RD. Overexpression of glutathione S-transferase/glutathione peroxidase enhances the growth of transgenic tobacco seedlings during stress. Nat Biotechnol 1997;15:988-991.

22. Jouve L, Hoffmann L, Hausman JF. Polyamine, carbohydrate, and proline content changes during salt stress exposure of aspen (Populus tremula L.): involvement of oxidation and osmoregulation metabolism. Plant Biol (Stuttg) 2004;6:74-80.
23. Wendler C, Barniske M, Wild A. Effect of phosphinothricin (glufosinate) on photosynthesis and photorespiration of C3 and C4 plants. Photosynth Res 1990;24:55-61.

24. Pascual MB. Expresio'n de glutamina sintetasa e isocitrato deshidrogenasa en a'rboles transge' nicos: papel en el desarrollo [dissertation]. Malaga: Universidad de Malaga, 2007. 